\title{
Retinal sensitivity after resolution of the macular edema associated with retinal vein occlusion.
}

\section{$\operatorname{AUTHOR}(\mathrm{S}):$}

Ota, Masafumi; Tsujikawa, Akitaka; Ojima, Yumiko; Miyamoto, Kazuaki; Murakami, Tomoaki; Ogino, Ken; AkagiKurashige, Yumiko; Muraoka, Yuki; Yoshimura, Nagahisa

\section{CITATION:}

Ota, Masafumi ...[et al]. Retinal sensitivity after resolution of the macular edema associated with retinal vein occlusion.. Graefe's archive for clinical and experimental ophthalmology 2012, 250(5): 635-644

\section{ISSUE DATE:}

2012-05

URL:

http://hdl.handle.net/2433/158358

\section{RIGHT:}

The final publication is available at www.springerlink.com; This is not the published version. Please cite only the published version.; この論文 は出版社版でありません。引用の際には出版社版をご確認ご利用くだ さい。 


\section{Title page}

\section{Retinal sensitivity after resolution of the macular edema associated with retinal} vein occlusion

Masafumi Ota, MD, Akitaka Tsujikawa, MD Yumiko Ojima, MD, Kazuaki Miyamoto, MD, Tomoaki Murakami, MD, Ken Ogino, MD, Yumiko Akagi-Kurashige, MD, Yuki Muraoka, MD, Nagahisa Yoshimura, MD

Department of Ophthalmology and Visual Sciences, Kyoto University Graduate School of Medicine, Kyoto, Japan.

15 Corresponding author: Akitaka Tsujikawa, MD, Department of Ophthalmology, Kyoto University Graduate School of Medicine, Sakyo-ku, Kyoto 606-8507, Japan. Tel: +81-75-751-3260; fax: +81-75-752-0933; e-mail: tujikawa@kuhp.kyoto-u.ac.jp.

The authors have no financial interest in the materials or devices mentioned in this 20 article. 
Microperimetry in Retinal Vein Occlusion. Ota et al. Page 2

\section{Abstract}

Purpose To study the correlation of retinal sensitivity with both morphologic changes in the macula and status of retinal capillary perfusion, after resolution of the macular edema associated with retinal vein occlusion (RVO).

Methods Retinal sensitivity in the macular area was examined with the Micro Perimeter 1 in 24 eyes after resolution of the macular edema associated with RVO. Using spectral-domain optical coherence tomography, $6 \mathrm{~mm} \times 6 \mathrm{~mm}$ areas of macula were examined with 256 sequential horizontal scans. Condition of the photoreceptor layer was evaluated depending upon detection of the junctions between inner and outer segments of the photoreceptors (IS/OS). Fluorescein angiography was performed in 19 eyes.

Results Mean retinal sensitivity on the affected side of the retina was significantly decreased $(p<0.001)$. On the affected side, the mean retinal sensitivity within the area of deteriorated IS/OS was significantly less $(3.8 \pm 4.8 \mathrm{~dB})$ than that within areas with

15 complete IS/OS $(10.1 \pm 6.4 \mathrm{~dB}, p<0.001)$. Mean retinal sensitivity within nonperfused areas was extremely low $(0.3 \pm 1.3 \mathrm{~dB})$, compared with that in perfused retina $(10.9 \pm 5.9$ $\mathrm{dB}, p<0.001)$. In eyes with a broken foveal capillary ring (FCR), the marked decline in retinal sensitivity was seen within the area where the FCR was broken; this was not seen in eyes with an intact FCR.

20 Conclusion Retinal function was decreased markedly in areas with a damaged photoreceptor layer due to RVO, and was lethally decreased within nonperfused areas. Due to the various limitations of the current study, including implementation of fluorescein angiography in limited number of eyes, wide range of follow-up, and heterogeneity of pretreatments, further prospective studies are necessary to confirm the current findings. 
Microperimetry in Retinal Vein Occlusion. Ota et al. Page 3

Key words fluorescein angiography, macular edema, microperimetry, optical coherence tomography, retinal vein occlusion. 


\section{Introduction}

Macular edema (ME) is one of the major vision-threatening complications associated with retinal vein occlusion (RVO) [1,2]. In RVO, increased intravascular pressure and reduced blood flow in the macular capillaries can lead to dysfunction of the endothelial blood-retinal barrier and to increased vascular permeability, both of which result in ME. To date, various treatments have been reported to successfully reduce retinal thickening and restore foveal function, which leads to improvement in visual acuity (VA) [1,3-21]. In the clinical setting, however, some patients have persistent symptoms, such as

10 metamorphopsia, blurred vision, and relative scotoma, despite complete resolution of their ME and good VA.

Optical coherence tomography (OCT) is currently widely used to obtain quantitative measurements of the foveal thickness and to detect morphologic changes in the retinal architecture. Due to its high resolution and contrast, OCT allows clinicians to use the

15 junctions between the inner and outer segments of the photoreceptors (IS/OS) as a hallmark of the integrity of the outer photoreceptor layer [22-25]. Thus, the condition of the IS/OS in the fovea is reported to be closely related to VA in eyes with various conditions, including both resolved and persistent ME associated with RVO [22-24]. Because VA reflects only foveal function, measurement of VA alone is not sufficient to evaluate visual function throughout the macular area after resolution of the ME associated with RVO.

Fundus-related microperimetry (Micro Perimeter 1 [MP1]; Nidek, Gamagori, Japan) can be used to obtain reliable quantitative measurements of retinal sensitivity in the macula by tracking eye movements [26-29]. With the use of the MP1, retinal sensitivity 
extrafoveal areas of eyes with ME associated with branch retinal vein occlusion (BRVO) or diabetic retinopathy [29-35]. Recently, Unoki et al. reported that retinal sensitivity as determined by the MP1 was severely decreased in capillary nonperfused areas (NPAs) of eyes with diabetic retinopathy [33]. However, little information is available on retinal function of the macula after complete resolution of the ME associated with RVO. This study was designed to examine retinal sensitivity using the MP1 in eyes with resolved ME secondary to RVO; this study was performed to determine any relationships between retinal sensitivity and morphologic changes in the retina, especially in its outer aspect, and with the status of 10 retinal capillary perfusion. 


\section{Materials and Methods}

Patient selection

5 We retrospectively reviewed the medical records of 30 patients (30 eyes) with resolved ME associated with RVO who were examined by the MP1 between May 2008 and June 2009. Eyes with central retinal vein occlusion were excluded from the current study. All eyes had shown marked ME at the initial visit, with foveal thickness greater than 300 $\mu \mathrm{m}$. Most eyes had undergone some surgical treatment, and all eyes showed complete

10 resolution of their ME at the time of inclusion in the current study. In this study, ME was considered to be resolved when foveal thickness was decreased to less than $250 \mu \mathrm{m}$ with no cystoid spaces in the macular area and when no recurrence of ME (foveal thickness equal to or greater than $250 \mu \mathrm{m}$, or the reappearance of cystoid spaces or subretinal fluid) was seen thereafter. Eyes with co-existing ocular disease (i.e.,

15 epiretinal membrane, glaucoma, diabetic retinopathy, or senile cataracts that resulted in poor-quality OCT images) were excluded from the current study. This study was approved by the Institutional Review Board at Kyoto University Graduate School of Medicine and adhered to the tenets of the Declaration of Helsinki.

All patients had undergone comprehensive ophthalmologic examinations, including best-corrected VA measurement, slit-lamp biomicroscopy, indirect fundus ophthalmoscopy, and OCT examination. Best-corrected VA was measured with a Landolt chart and converted to a logarithm of the minimal angle of resolution (logMAR). In each eye, the MP1 measurement was performed after resolution of the ME secondary 
to RVO. OCT examination was performed with the use of a spectral-domain OCT (3D-OCT 1000; Topcon, Tokyo, Japan) to evaluate morphologic changes of the retina in the larger macular area. Nineteen of the 24 patients underwent fluorescein angiography (FA) with a confocal laser scanning system (HRA-2; Heidelberg Engineering, Heidelberg, Germany) after resolution of the ME. In some cases, we used the data previously obtained by time-domain OCT (Stratus OCT 3000; Carl Zeiss, Dublin, California, USA).

MP1 measurements

10 Fundus-monitored microperimetry was performed with the MP1 in all eyes. The MP1 is equipped with an automatic tracking system that evaluates every acquired frame for shifts in the horizontal and vertical directions of the fundus with respect to a reference frame obtained by an infrared camera at the beginning of the examination. A 4-2-staircase strategy with Goldmann III size stimulus was used, and 57 stimulus

15 locations that covered the central 10 degrees were examined (Fig. 1). The white background illumination was set at $1.27 \mathrm{~cd} / \mathrm{m}^{2}$. The differential luminance, defined as the difference between stimulus luminance and background luminance, was $127 \mathrm{~cd} / \mathrm{m}^{2}$ at 0 decibel $(\mathrm{dB})$ stimulation, and the maximum stimulus attenuation was $20 \mathrm{~dB}$. The duration of the stimulus was $200 \mathrm{~ms}$, and the fixation target was varied in size according

20 to the patient's VA. Light stimuli were presented randomly during the examination, and results were reported in $\mathrm{dB}$. To more clearly depict the clinical correlation between data obtained by microperimetry and actual retinal features, functional results were displayed on a color digital retinograph.

In the current study, the macular area examined by the MP1 was divided into 3 25 regions: the foveal region, the affected side, and the unaffected side. The foveal region 
consisted of 9 stimulus locations that covered the central 2 degrees. Outside of the fovea, the macula was divided into 2 regions by a horizontal line that passed through the fovea. The affected side and unaffected side of the retina were determined by the presence of RVO; each side contained 24 stimulus locations (Fig. 1). Six eyes were excluded from the current study due to poor fixation or extrafoveal fixation.

\section{D-OCT examination}

After complete resolution of edema, the macular area of each eye was examined with 3D-OCT. A $6 \times 6-\mathrm{mm}$ area of the macular region centered on the fovea was examined with 256 sequential horizontal scans. Each horizontal scan consisted of 256 axial scans. In each eye, the retinal structure of this $6 \times 6-\mathrm{mm}$ macular area was evaluated by observation of each OCT scan. In most scans, the IS/OS lines appeared to be intact, but some sections showed a focal defect of the IS/OS reflective line. The OCT

15 evaluations were done by a fully trained ophthalmologist (Y.O.) who had no knowledge of the VA or any other conditions of the eyes; a sequence of OCT scans from each data set was examined, and areas with a deteriorated IS/OS were identified on the fundus projection images.

20 Comparison of the results (MP1, 3D-OCT, and FA)

After comparing the areas with deteriorated or intact IS/OS, which were determined by 3D-OCT, with the MP1 results, we noted the retinal sensitivity and condition of the IS/OS at each stimulus location in the MP1 examination of each eye. The fundus projection 
sensitivity map obtained with the MP1 and the condition of IS/OS was determined at each measurement point. In addition, in 19 of the 24 eyes on which FA was performed, superimposing the MP1 data on the FA image was done to study the correlation between the MP1 results and FA findings. After both the MP1 and FA image had been magnified

5 (Fig. 2), the overlay was done by matching the fovea and 2 arteriovenous crossings, which were located on opposite sides of the fovea. Based on this composite image, we noted retinal sensitivity and status of the retinal perfusion (perfusion or nonperfusion) at each stimulus location in the MP1 examination of each eye.

10 Statistical analysis

Statistical analysis was performed using a software designed for this purpose (StatView, version 5.0; SAS Institute, Cary, North Carolina, USA). All values are presented as mean \pm standard deviation. The retinal sensitivity obtained with MP1 was compared

15 with that determined from the Mann-Whitney $U$ test due to its non-normal distribution. The Student's $t$ test was used for comparison of foveal thickness and age. Categorical analysis was performed using the Chi-square test. A $p$ value of less than 0.05 was considered to be statistically significant. 


\section{Results}

In the current study, we examined 24 eyes of 24 patients (10 men and 14 women) who experienced resolution of the ME associated with RVO; the patients ranged in age from 47 to 85 years $(66.8 \pm 10.1$ years). Table 1 shows the clinical characteristics of all 24 patients. At the initial visit, all eyes showed marked ME and decreased VA associated with acute BRVO (17 eyes) or acute hemi-central vein occlusion (CRVO) ( 7 eyes). The mean initial VA was $0.49 \pm 0.33$ (in logMAR), and the mean initial foveal thickness was $554 \pm 97 \mu \mathrm{m}$. Treatments for acute ME consisted of intravitreal injection of

10 bevacizumab in 16 eyes, sub-tenon injection of triamcinolone acetonide in 2 eyes, pars plana vitrectomy in 3 eyes, and grid laser photocoagulation in 5 eyes. No surgical treatment was performed in 2 eyes. After the ME had completely resolved, MP1 measurement was performed for each patient. Mean VA at that time was $0.07 \pm 0.16$ (in $\log M A R$ ), and mean foveal thickness was $210 \pm 30 \mu \mathrm{m}$. The mean duration from the

15 final resolution of ME to the MP1 and OCT examinations was $11.5 \pm 6.5$ months (3-24 months).

MP1 examination showed that retinal sensitivity was markedly decreased on the affected side of the retina compared with the unaffected side. In 1152 stimulus locations of 24 eyes (576 on the affected side and 576 on the unaffected side), excluding

20 the foveal region, the mean retinal sensitivity on the affected side $(8.9 \pm 6.6 \mathrm{~dB})$ was significantly less than that on the unaffected side $(15.5 \pm 3.5, p<0.001)$.

The condition of the IS/OS at each stimulus location was determined from a sequence of OCT sections (Table 2). On the unaffected side, only $28(4.9 \%)$ of a total of 576 points showed deterioration of the IS/OS. On the affected side, however, as 
many as 110 points (19.1\%) showed deterioration of the IS/OS, and the number of points at which the IS/OS was deteriorated was significantly greater on the affected side than on the unaffected side $(p<0.001)$. Retinal sensitivity was focally decreased in the areas with deteriorated IS/OS (Fig. 3). Mean retinal sensitivity was the highest in stimulus locations on the unaffected side with complete IS/OS, while areas with deteriorated IS/OS on the affected side showed the lowest sensitivity. On the affected side of the retina, the mean retinal sensitivity at the stimulus locations with deteriorated IS/OS (3.8 \pm $4.8 \mathrm{~dB}$ ) was significantly less than that at stimulus locations with complete IS/OS (10.1 \pm $6.4 \mathrm{~dB}, p<0.001)$.

FA was performed in 19 of the 24 eyes; we studied capillary perfusion status at a total of 456 stimulus locations on the affected side of the retina (24 locations in each eye) (Fig. 2). Of these 456 locations, 102 were in the nonperfused area and the remaining 354 were in the perfused area (Table 3). In the perfused area, retinal sensitivity varied from $0 \mathrm{~dB}$ to $20 \mathrm{~dB}$, although the mean retinal sensitivity at locations with deteriorated

15 IS/OS was significantly less than that in locations with intact IS/OS $(p<0.001)$. In contrast, retinal sensitivity was severely decreased in the nonperfused area (Fig. 4). Mean retinal sensitivity at the locations in the nonperfused region was extremely low $(0.3$ $\pm 1.3 \mathrm{~dB})$, compared with that in the perfused area $(10.9 \pm 5.9 \mathrm{~dB}, p<0.001)$. More specifically, all 28 locations with deteriorated IS/OS in the nonperfused area showed a value of $0 \mathrm{~dB}$. In nonperfused areas, the stimulus locations, even those with intact IS/OS, showed significantly less sensitivity $(0.5 \pm 1.5 \mathrm{~dB})$ than did points in the perfused area with deteriorated IS/OS $(5.1 \pm 4.9 \mathrm{~dB}, p<0.001$, Fig. 5).

The condition of the foveal capillary ring (FCR) was evaluated in 19 eyes. Of these 19 eyes, 10 had a broken FCR, and it was intact in the other 9 eyes (Table 4). In 
groups $(0.04 \pm 0.21$ in eyes with an intact FCR and $0.15 \pm 0.12$ in eyes with a broken FCR). No statistically significant difference was found between these groups in the initial VA $(p=0.13)$ or VA at resolution of the ME $(p=0.055)$, which tended to be better in eyes with an intact FCR than in those with a broken FCR. However, mean retinal sensitivity within the foveal region (within the central 2 degrees) was significantly poorer in eyes with a broken FCR $(10.9 \pm 6.5 \mathrm{~dB})$ than in those with an intact FCR $(13.7 \pm 4.9 \mathrm{~dB}$, $p<0.001)$. In eyes with a broken FCR, a marked decline in retinal sensitivity was seen in the area where the FCR was broken (Fig. 4), but was not seen in eyes with an intact FCR. 


\section{Discussion}

Various treatments are reportedly effective in reducing the ME associated with RVO, such as grid laser photocoagulation [1,3-5], pars plana vitrectomy [6-8], and intravitreal

5 injections of triamcinolone acetonide [8-14] or bevacizumab [15-21]. After successful treatment, resolution of the ME often leads to substantial improvement of VA. In most reports, the efficacy of these treatments was evaluated based on a change in the foveal thickness as measured by OCT and according to measurement of VA. However, VA reflects only foveal function, and because most of the ME associated with RVO encompasses a larger area, VA measurement does not necessarily reflect true loss of VA. In the clinical setting, even after complete resolution of the ME is obtained, some patients have persistent symptoms, such as metamorphopsia, blurred vision, and scotoma. Therefore, evaluation of the larger macular area is valuable to better determine visual function after resolution of the ME associated with RVO.

In the present study, we first investigated the correlation between retinal sensitivity and condition of the IS/OS in the macular area after resolution of the ME. On the unaffected side of the retina, $95 \%$ of the locations showed intact IS/OS; however, $19 \%$ of locations on the affected side of the retina showed deteriorated IS/OS. Retinal ischemia and swelling in the photoreceptor layer due to acute RVO results in deterioration of the photoreceptor cells, which is detected as the deteriorated IS/OS on OCT. Stimulus locations in areas with deteriorated IS/OS showed significantly less retinal sensitivity than did those with intact IS/OS, so the structural abnormality of the photoreceptor layer in eyes with resolved ME causes the focal decrease in retinal function within the larger macular area. 
chorioretinopathy, a defect of the IS/OS showed a focal decrease in retinal sensitivity [26]. They speculated that this defect might account for the persistent visual disturbance after complete resolution of the retinal detachment was obtained. Routine VA measurement does not reflect these small structural and functional abnormalities, even if the patient has persistent symptoms. In the current study, we made similar observations in eyes after resolution of the ME associated with RVO. Focal damage of the photoreceptor layer, which was detected as deterioration of the IS/OS, may partially account for the persistent visual disturbance after resolution of the ME and return of good VA.

In addition, we investigated the relationship between retinal sensitivity and retinal capillary perfusion. Mean retinal sensitivity within the nonperfused area was substantially reduced to $0.3 \pm 1.3 \mathrm{~dB}$, which was markedly less than that in the perfused area $(10.9 \pm 5.9 \mathrm{~dB}) . \quad$ In nonperfused areas, the stimulus locations, even those with intact IS/OS, had only $0.5 \pm 1.5 \mathrm{~dB}$ retinal sensitivity, while all the locations with deteriorated IS/OS had a retinal sensitivity of $0 \mathrm{~dB}$ (absolute scotoma). With the use of

15 the Humphrey field analyzer, Chee and Flanagan previously reported reduced retinal sensitivity within areas of capillary nonperfusion in diabetic retinopathy [36]. In eyes with diabetic retinopathy and those with acute BRVO, Bell and Feldon reported a good correspondence between regional visual field sensitivities and retinal capillary perfusion [37]. Our finding is consistent with these previous reports: retinal sensitivity in nonperfused areas was extremely low even after complete resolution of both the retinal hemorrhage and the edema.

In eyes with diabetic retinopathy, Unoki et al. recently have shown that the inner retina was thinner and more disorganized in nonperfused areas than in perfused retinal areas [33]. The inner retina would be particularly at risk to hypoxic insult from RVO 
relatively sparse compared with choroidal circulation. In the current study, as shown in Figure 4, thinning and disorganization in the inner retina were seen within areas of nonperfusion, even if the outer retina appeared almost intact. As discussed above, damage to the outer photoreceptor layer results in a moderate decrease in retinal

5 function. However, damage to the inner retina due to capillary nonperfusion causes a marked decrease in retinal function, which often leads to an absolute scotoma.

Previously, Finkelstein reported that eyes with ischemic ME caused by BRVO had a better visual prognosis than did those with perfused ME, and indicated that a broken FCR is frequently seen in ischemic ME [38]. However, other reports showed that BRVO

10 accompanied by a broken FCR indicated a poor prognosis for VA [39-41], so the association between FCR status and VA remains controversial. In the current study, eyes with either a broken or intact FCR achieved relatively good VA after the resolution of $M E$, and there were no statistically significant differences in VA. However, mean foveal sensitivity of eyes with a broken FCR was significantly lower than that for eyes with an

15 intact FCR. In eyes with a broken FCR, marked foveal sensitivity loss was shown regionally to be consistent with the break in the FCR. In the current study, we cannot make any conclusions regarding an association between FCR status and visual prognosis, although we do suggest that eyes with a broken FCR have significant loss of retinal sensitivity in the parafoveal region, which might cause some visual disturbance

20 after resolution of the ME, even if complete recovery of VA is achieved.

One limitation of the current study is its small sample size. To evaluate retinal function, we separated the macular area into an affected side and an unaffected side. However, this separation does not accurately reflect the area affected by RVO because of variations in retinal vasculature. Our findings do suggest, however, that retinal 
to RVO. However, retinal function within nonperfused areas was lethally decreased, even in eyes in which the outer retina was well preserved. Moreover, we demonstrated that eyes with a broken FCR showed a marked loss of sensitivity in the area corresponding to the break in the FCR. Such factors may be associated with persistent 5 symptoms, such as blurred vision or relative scotoma, even after complete resolution of the ME and good recovery of VA.

Other limitations of the current study are the difference in the treatment regimens used and the wide range of the follow-up period. Treatment and the duration of ME may influence the perfusion status or integrity of the photoreceptor layer. In the current study,

10 however, retinal function, integrity of the photoreceptor layer, and retinal perfusion status were all evaluated after complete resolution of the ME. Further prospective studies are necessary to elucidate the effect of treatment and the duration of ME on retinal function and these pathologic conditions associated with RVO. 


\section{References}

1. The Branch Vein Occlusion Study Group (1984) Argon laser photocoagulation for macular edema in branch vein occlusion. Am J Ophthalmol 98:271-282

5 2. Glacet-Bernard A, Coscas G, Chabanel A, Zourdani A, Lelong F, Samama MM (1996) Prognostic factors for retinal vein occlusion: prospective study of 175 cases. Ophthalmology 103:551-560

3. Arnarsson A, Stefánsson E (2000) Laser treatment and the mechanism of edema reduction in branch retinal vein occlusion. Invest Ophthalmol Vis Sci 41:877-879

10 4. Esrick E, Subramanian ML, Heier JS, Devaiah AK, Topping TM, Frederick AR, Morley MG (2005) Multiple laser treatments for macular edema attributable to branch retinal vein occlusion. Am J Ophthalmol 139:653-657

5. Ohashi H, Oh H, Nishiwaki H, Nonaka A, Takagi H (2004) Delayed absorption of macular edema accompanying serous retinal detachment after grid laser treatment in patients with branch retinal vein occlusion. Ophthalmology $111: 2050-2056$

6. Horio N, Horiguchi M (2005) Effect of arteriovenous sheathotomy on retinal blood flow and macular edema in patients with branch retinal vein occlusion. Am J Ophthalmol 139:739-740

$207 . \quad$ Mandelcorn MS, Nrusimhadevara RK (2004) Internal limiting membrane peeling for decompression of macular edema in retinal vein occlusion: a report of 14 cases. Retina 24:348-355

8. Tsujikawa A, Fujihara M, Iwawaki T, Yamamoto K, Kurimoto Y (2005) Triamcinolone acetonide with vitrectomy for treatment of macular edema associated with branch retinal vein occlusion. Retina 25:861-867 
9. Cekic O, Chang S, Tseng JJ, Barile GR, Del Priore LV, Weissman H, Schiff WM, Ober MD (2005) Intravitreal triamcinolone injection for treatment of macular edema secondary to branch retinal vein occlusion. Retina 25:851-855

10. Chen SD, Sundaram V, Lochhead J, Patel CK (2006) Intravitreal triamcinolone for the treatment of ischemic macular edema associated with branch retinal vein occlusion. Am J Ophthalmol 141:876-883

11. Goff MJ, Jumper JM, Yang SS, Fu AD, Johnson RN, McDonald HR, Ai E (2006) Intravitreal triamcinolone acetonide treatment of macular edema associated with central retinal vein occlusion. Retina 26:896-901

12. Karacorlu M, Ozdemir H, Karacorlu SA (2005) Resolution of serous macular detachment after intravitreal triamcinolone acetonide treatment of patients with branch retinal vein occlusion. Retina 25:856-860

13. Ozdek SC, Aydin B, Gürelik G, Bahceci U, Hasanreisoğlu B (2005) Effects of intravitreal triamcinolone injection on macular edema and visual prognosis in central retinal vein occlusion. Int Ophthalmol 26:27-34

14. Williamson TH, O'Donnell A (2005) Intravitreal triamcinolone acetonide for cystoid macular edema in nonischemic central retinal vein occlusion. Am J Ophthalmol $139: 860-866$

15. Hsu J, Kaiser RS, Sivalingam A, Abraham P, Fineman MS, Samuel MA, Vander JF, Regillo CD, Ho AC (2007) Intravitreal bevacizumab (Avastin) in central retinal vein occlusion. Retina 27:1013-1019

16. Iturralde D, Spaide RF, Meyerle CB, Klancnik JM, Yannuzzi LA, Fisher YL, Sorenson J, Slakter JS, Freund KB, Cooney M, Fine HF (2006) Intravitreal bevacizumab (Avastin) treatment of macular edema in central retinal vein occlusion: a short-term study. Retina 26:279-284 
17. Jaissle GB, Leitritz M, Gelisken F, Ziemssen F, Bartz-Schmidt KU, Szurman P (2009) One-year results after intravitreal bevacizumab therapy for macular edema secondary to branch retinal vein occlusion. Graefe's Arch Clin Exp Ophthalmol 247:27-33

18. Kreutzer TC, Alge CS, Wolf AH, Kook D, Burger J, Strauss R, Kunze C, Haritoglou C, Kampik A, Priglinger S (2008) Intravitreal bevacizumab for the treatment of macular oedema secondary to branch retinal vein occlusion. Br J Ophthalmol $92: 351-355$

19. Kriechbaum K, Michels S, Prager F, Georgopoulos M, Funk M, Geitzenauer W, Schmidt-Erfurth U (2008) Intravitreal Avastin for macular oedema secondary to retinal vein occlusion: a prospective study. Br J Ophthalmol 92:518-522

20. Pai SA, Shetty R, Vijayan PB, Venkatasubramaniam G, Yadav NK, Shetty BK, Babu RB, Narayana KM (2007) Clinical, anatomic, and electrophysiologic evaluation following intravitreal bevacizumab for macular edema in retinal vein occlusion. Am J Ophthalmol 143:601-606

21. Rosenfeld PJ, Fung AE, Puliafito CA (2005) Optical coherence tomography findings after an intravitreal injection of bevacizumab (Avastin) for macular edema from central retinal vein occlusion. Ophthalmic Surg Lasers Imaging 36:336-339

22. Ota M, Tsujikawa A, Kita M, Miyamoto K, Sakamoto A, Yamaike N, Kotera Y, Yoshimura N (2008) Integrity of foveal photoreceptor layer in central retinal vein occlusion. Retina 28:1502-1508

23. Ota M, Tsujikawa A, Murakami T, Kita M, Miyamoto K, Sakamoto A, Yamaike N, Yoshimura N (2007) Association between integrity of foveal photoreceptor layer and visual acuity in branch retinal vein occlusion. Br J Ophthalmol 91:1644-1649

25 24. Ota M, Tsujikawa A, Murakami T, Yamaike N, Sakamoto A, Kotera Y, Miyamoto K, 
Kita M, Yoshimura N (2008) Foveal photoreceptor layer in eyes with persistent cystoid macular edema associated with branch retinal vein occlusion. Am J Ophthalmol 145:273-280

25. Yamaike N, Tsujikawa A, Ota M, Sakamoto A, Kotera Y, Kita M, Miyamoto K, Yoshimura N, Hangai M (2008) Three-dimensional imaging of cystoid macular edema in retinal vein occlusion. Ophthalmology 115:355-362 e352

26. Ojima Y, Tsujikawa A, Hangai M, Nakanishi H, Inoue R, Sakamoto A, Yoshimura N (2008) Retinal sensitivity measured with the micro perimeter 1 after resolution of central serous chorioretinopathy. Am J Ophthalmol 146:77-84

10 27. Rohrschneider K, Springer C, Bültmann S, Völcker HE (2005) Microperimetry--comparison between the micro perimeter 1 and scanning laser ophthalmoscope--fundus perimetry. Am J Ophthalmol 139:125-134

28. Springer C, Bültmann S, Völcker HE, Rohrschneider K (2005) Fundus perimetry with the Micro Perimeter 1 in normal individuals: comparison with conventional threshold perimetry. Ophthalmology 112:848-854

29. Yamaike N, Tsujikawa A, Sakamoto A, Ota M, Kotera Y, Miyamoto K, Kita M, Yoshimura N (2009) Retinal sensitivity after intravitreal injection of bevacizumab for the treatment of macular edema secondary to retinal vein occlusion. Retina $29: 757-767$

20 30. Imasawa M, lijima H, Morimoto T (2001) Perimetric sensitivity and retinal thickness in eyes with macular edema resulting from branch retinal vein occlusion. Am J Ophthalmol 131:55-60

31. Kriechbaum K, Prager F, Geitzenauer W, Benesch T, Schutze C, Simader C, Schmidt-Erfurth U (2009) Association of retinal sensitivity and morphology during antiangiogenic treatment of retinal vein occlusion over one year. Ophthalmology 
Microperimetry in Retinal Vein Occlusion. Ota et al. Page 21

$116: 2415-2421$

32. Okada K, Yamamoto S, Mizunoya S, Hoshino A, Arai M, Takatsuna Y (2006) Correlation of retinal sensitivity measured with fundus-related microperimetry to visual acuity and retinal thickness in eyes with diabetic macular edema. Eye (Lond) 20:805-809

33. Unoki N, Nishijima K, Sakamoto A, Kita M, Watanabe D, Hangai M, Kimura T, Kawagoe N, Ohta M, Yoshimura N (2007) Retinal sensitivity loss and structural disturbance in areas of capillary nonperfusion of eyes with diabetic retinopathy. Am J Ophthalmol 144:755-760

10 34. Vujosevic S, Midena E, Pilotto E, Radin PP, Chiesa L, Cavarzeran F (2006) Diabetic macular edema: correlation between microperimetry and optical coherence tomography findings. Invest Ophthalmol Vis Sci 47:3044-3051

35. Yamaike N, Kita M, Tsujikawa A, Miyamoto K, Yoshimura N (2007) Perimetric sensitivity with the Micro Perimeter 1 and retinal thickness in patients with branch retinal vein occlusion. Am J Ophthalmol 143:342-344

36. Chee CK, Flanagan DW (1993) Visual field loss with capillary non-perfusion in preproliferative and early proliferative diabetic retinopathy. $\mathrm{Br} \mathrm{J}$ Ophthalmol $77: 726-730$

37. Bell JA, Feldon SE (1984) Retinal microangiopathy. Correlation of OCTOPUS perimetry with fluorescein angiography. Arch Ophthalmol 102:1294-1298

38. Finkelstein D (1992) Ischemic macular edema. Recognition and favorable natural history in branch vein occlusion. Arch Ophthalmol 110:1427-1434

39. Chung EJ, Hong YT, Lee SC, Kwon OW, Koh HJ (2008) Prognostic factors for visual outcome after intravitreal bevacizumab for macular edema due to branch retinal vein occlusion. Graefes Arch Clin Exp Ophthalmol 246:1241-1247 
40. Clemett RS, Kohner EM, Hamilton AM (1973) The visual prognosis in retinal branch vein occlusion. Trans Ophthalmol Soc U K 93:523-535

41. Shilling JS, Jones CA (1984) Retinal branch vein occlusion: a study of argon laser photocoagulation in the treatment of macular oedema. $\mathrm{Br} \mathrm{J}$ Ophthalmol 68:196-198 


\section{Figure legends}

Fig. 1. Micro Perimeter 1 image shows retinal sensitivity at each stimulus site in decibels. Fifty-seven stimulus points are located in the macular area covering the central 10 degrees, which is divided into three regions; the foveal region (central circle) consists of nine stimulus locations which covers the central 2 degrees and a minor region outside the fovea, the affected side, and the side unaffected by retinal vein occlusion, the latter two of which each consist of 24 stimulus locations.

10 Fig. 2. Fluorescein angiogram and Micro Perimeter 1 (MP1) image of an eye with branch retinal vein occlusion. a Fluorescein angiogram shows an area of capillary nonperfusion and scars from the grid laser photocoagulation in the superior-temporal area of the macula. b MP1 image shows that many 0 decibel $(\mathrm{dB})$ points are concentrated in the superior-temporal area of the macula. c MP1 results are

15 superimposed onto the fluorescein angiogram on the basis of the following points: the fovea and two arteriovenous crossings that are located on opposite sides of the fovea. Based on this composite image, we can identify the retinal sensitivity and the status of retinal perfusion (perfusion versus nonperfusion) of each stimulus location. In this particular case, the composite image shows the area of $0 \mathrm{~dB}$ to be well correlated with the area of capillary nonperfusion.

Fig. 3. A 65-year-old man had a six-week history of decreased visual acuity (VA) in the left eye ( 0.15 on Landolt chart) due to macular edema (ME) associated with hemi central retinal vein occlusion. a Fundus photograph at the initial visit shows extensive retinal hemorrhage. b The ME was resolved with intravitreal injections of bevacizumab. At 13 
months, VA in this eye had recovered to 1.2 on Landolt chart and foveal thickness was $215 \mu \mathrm{m}$. c Fluorescein angiogram shows minimal leakage from the retinal capillaries. d Sectional images obtained by optical coherence tomography (OCT) were made along long arrows shown in the retinal sensitivity map. OCT sections show small defects of

$5 \quad$ IS/OS (white arrows). OCT images show a mostly intact inner and outer retina. e Retinal sensitivity map obtained with the Micro Perimeter 1 shows focal areas with decreased sensitivity, consistent with the deteriorated IS/OS observed on the OCT images.

10 Fig. 4. A 69-year-old man had a one-week history of decreased visual acuity (VA) in the right eye ( 0.4 on Landolt chart) due to macular edema (ME) associated with hemi central retinal vein occlusion. a Fundus photograph at the initial visit shows extensive retinal hemorrhage. The ME was resolved with the treatment by intravitreal injections of bevacizumab. b At 22 months after the initial visit, VA in this eye was 0.6 on Landolt

15 chart and foveal thickness was $213 \mu \mathrm{m}$. c Fluorescein angiogram shows a large area of capillary nonperfusion (NPA), which involves the foveal region. d, Sectional images with optical coherence tomography (OCT) were obtained along the long arrows shown in the retinal sensitivity map. An OCT image in the perfused region of the retina (first row) shows intact structure of the retina. OCT image in NPA (fourth row) shows thinning and disorganization of the inner retina (black arrows) and relatively well preserved outer retina. e Retinal sensitivity map obtained with the Micro Perimeter 1 shows markedly decreased retinal sensitivity in the inferior macular area, consistent with the NPA detected by fluorescein angiogram.

25 Fig. 5. Histograms showing the numbers of measurement points with various retinal 
sensitivities measured with the Micro Perimeter 1 on the side of the macula affected by retinal vein occlusion. a Measurement points located in the area of capillary nonperfusion, which show the intact junctions between inner and outer segments of the photoreceptors (IS/OS). $\quad$ b Measurement points located in the perfused area, which also

5 show deterioration of the IS/OS. c Measurement points located in the area of capillary nonperfusion, showing deteriorated IS/OS. d Measurement points located in the perfused area, showing intact IS/OS. 
Table 1 Clinical characteristics of patients after resolution of macular edema associated with retinal vein occlusion

\begin{tabular}{lc}
\hline Number of patients and eyes & 24 (24 eyes) \\
Age (years) & $66.8 \pm 10.1$ \\
Gender (men/women) & $10 / 14$ \\
Hypertension (number of patients) & $19 / 5$ \\
Lens status (phakia/pseudophakia) & $0.49 \pm 0.33$ \\
Initial visual acuity (logMAR) & $0.07 \pm 0.16$ \\
Visual acuity at time of MP1 measurement (logMAR) & $554 \pm 97$ \\
Initial foveal thickness ( $\mu$ m) & $210 \pm 30$ \\
Foveal thickness at time of MP1 measurement ( $\mu$ ) & $6.7 \pm 4.8$ \\
Duration of macular edema (months) & $11.5 \pm 6.5$ \\
Duration from resolution of macular edema to MP1 measurement (months) \\
$\begin{array}{l}\text { Duration of follow-up (months) } \\
\text { Type of retinal vein occlusion (number of eyes) }\end{array}$ \\
$\begin{array}{l}\text { Branch retinal vein occlusion } \\
\text { HogMAR = logarithm of the minimum angle of resolution; MP1 = Micro Perimeter } 1\end{array}$ \\
$\begin{array}{l}\text { Duration of macular edema was defined as the duration from the initial symptom to the initial } \\
\text { resolution of macular edema. }\end{array}$ \\
\hline 26.7
\end{tabular}


Table 2 Condition of IS/OS and retinal sensitivity of eyes after resolution of macular edema associated with retinal vein occlusion

\begin{tabular}{lll}
\hline Retinal area & Number of points & Retinal sensitivity $(\mathrm{dB})$
\end{tabular}

Unaffected side

Whole

576

$15.5 \pm 3.5$

Complete IS/OS

548

$15.7 \pm 3.3$

Deteriorated IS/OS

28

$11.1 \pm 3.8$

\section{Affected side}

Whole

Complete IS/OS

Deteriorated IS/OS
576

466

110
$8.9 \pm 6.6^{*}$

$10.1 \pm 6.4^{\dagger}$

$3.8 \pm 4.8^{\ddagger \S}$

$\mathrm{IS} / \mathrm{OS}=$ junction between photoreceptor inner and outer segments; $\mathrm{dB}=$ decibel ${ }^{*} p<0.001$, compared with values of whole points on unaffected side.

${ }^{\dagger} p<0.001$, compared with values of complete IS/OS points on unaffected side.

${ }^{\ddagger} p<0.001$, compared with values of deteriorated IS/OS points on unaffected side.

${ }^{\S} p<0.001$, compared with values of complete IS/OS points on affected side. 
Table 3 Condition of IS/OS, retinal sensitivity and retinal perfusion status of eyes after resolution of macular edema associated with retinal vein occlusion

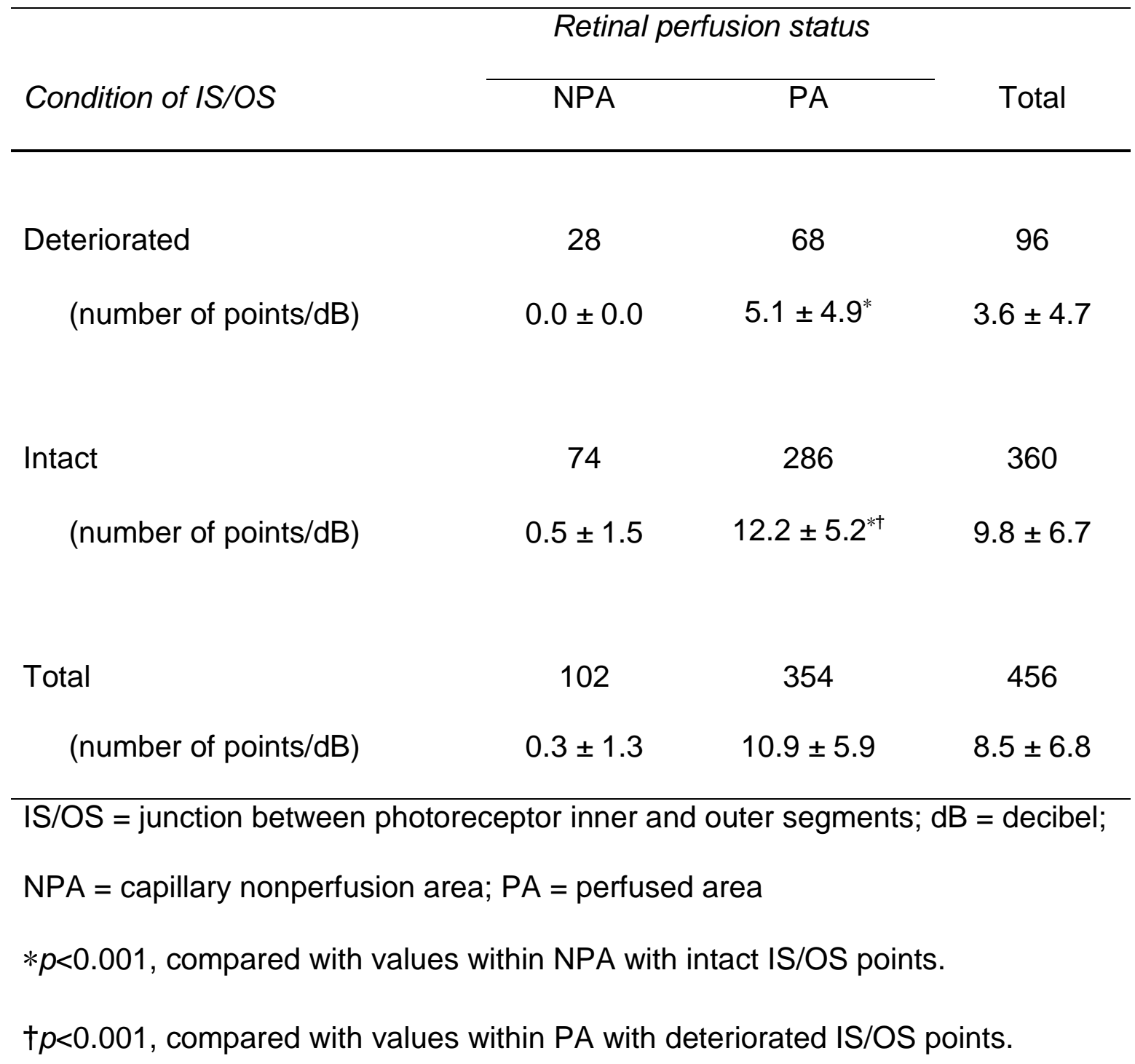


Table 4 Retinal sensitivity in the foveal region of eyes with broken or intact foveal capillary ring

\begin{tabular}{|c|c|c|c|}
\hline & $\begin{array}{l}\text { Eyes with } \\
\text { broken FCR }\end{array}$ & $\begin{array}{l}\text { Eyes with } \\
\text { intact FCR }\end{array}$ & $P$ value \\
\hline Number of eyes & 10 & 9 & - \\
\hline Age (years) & $65.7 \pm 13.0$ & $67.9 \pm 9.7$ & 0.57 \\
\hline Initial visual acuity (logMAR) & $0.69 \pm 0.33$ & $0.38 \pm 0.29$ & 0.13 \\
\hline Initial foveal thickness $(\mu \mathrm{m})$ & $561 \pm 106$ & $551 \pm 106$ & 0.85 \\
\hline $\begin{array}{l}\text { Visual acuity after resolution of } \\
\text { macular edema (logMAR) }\end{array}$ & $0.15 \pm 0.12$ & $0.04 \pm 0.21$ & 0.055 \\
\hline $\begin{array}{l}\text { Foveal thickness after resolution of } \\
\text { macular edema }(\mu \mathrm{m})\end{array}$ & $226 \pm 33$ & $199 \pm 22$ & 0.066 \\
\hline \multicolumn{4}{|l|}{$\begin{array}{l}\text { Type of retinal vein occlusion } \\
\text { (number of eyes) }\end{array}$} \\
\hline Branch retinal vein occlusion & 6 & 7 & \\
\hline Hemi central retinal vein occlusion & 4 & 2 & 0.41 \\
\hline Retinal sensitivity in foveal region $(\mathrm{dB})$ & $10.9 \pm 6.5$ & $13.7 \pm 4.9$ & $<0.001$ \\
\hline
\end{tabular}




\section{affected side}

foveal region ${ }^{\prime 2}$ 10 


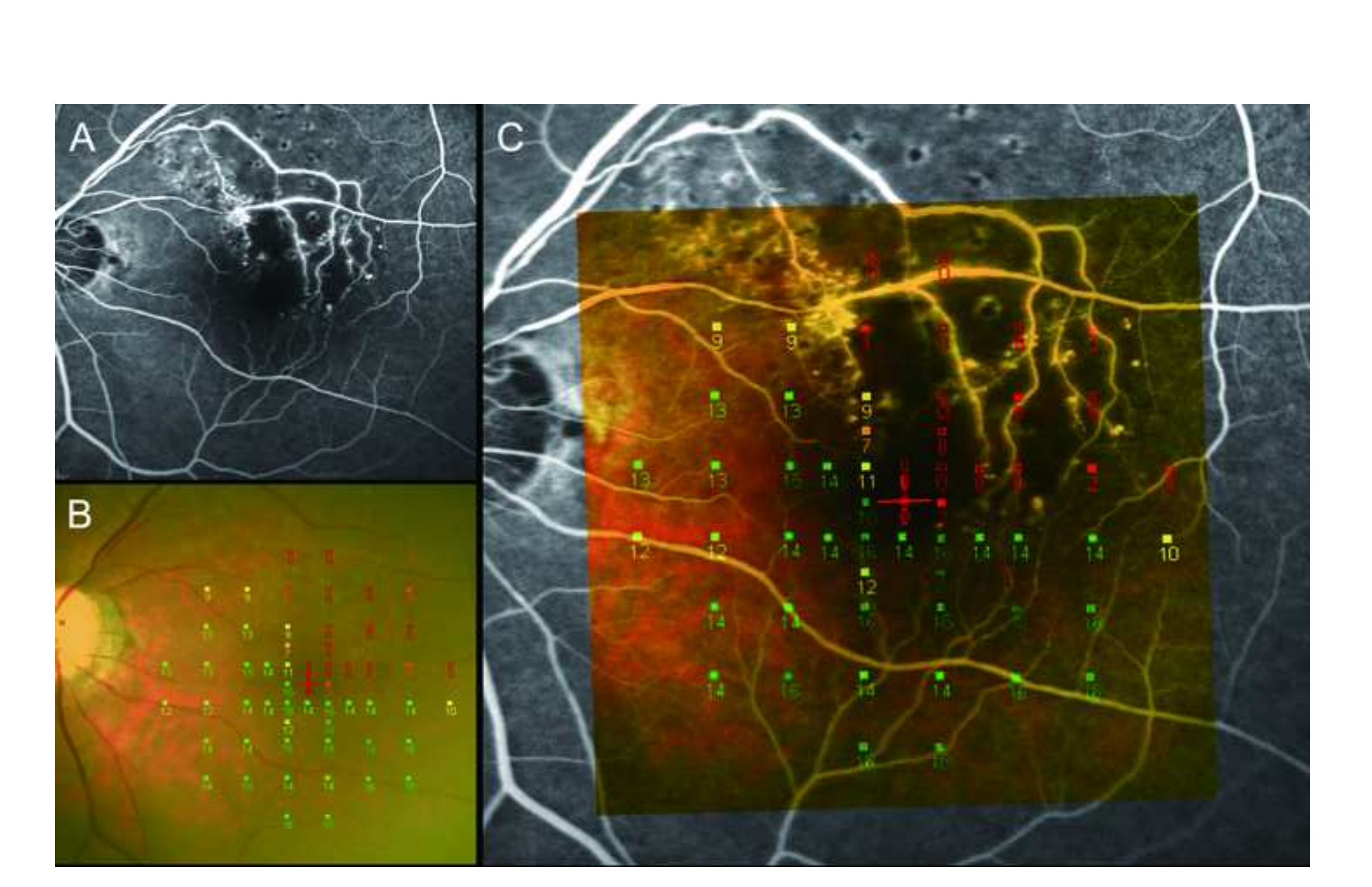

-

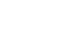

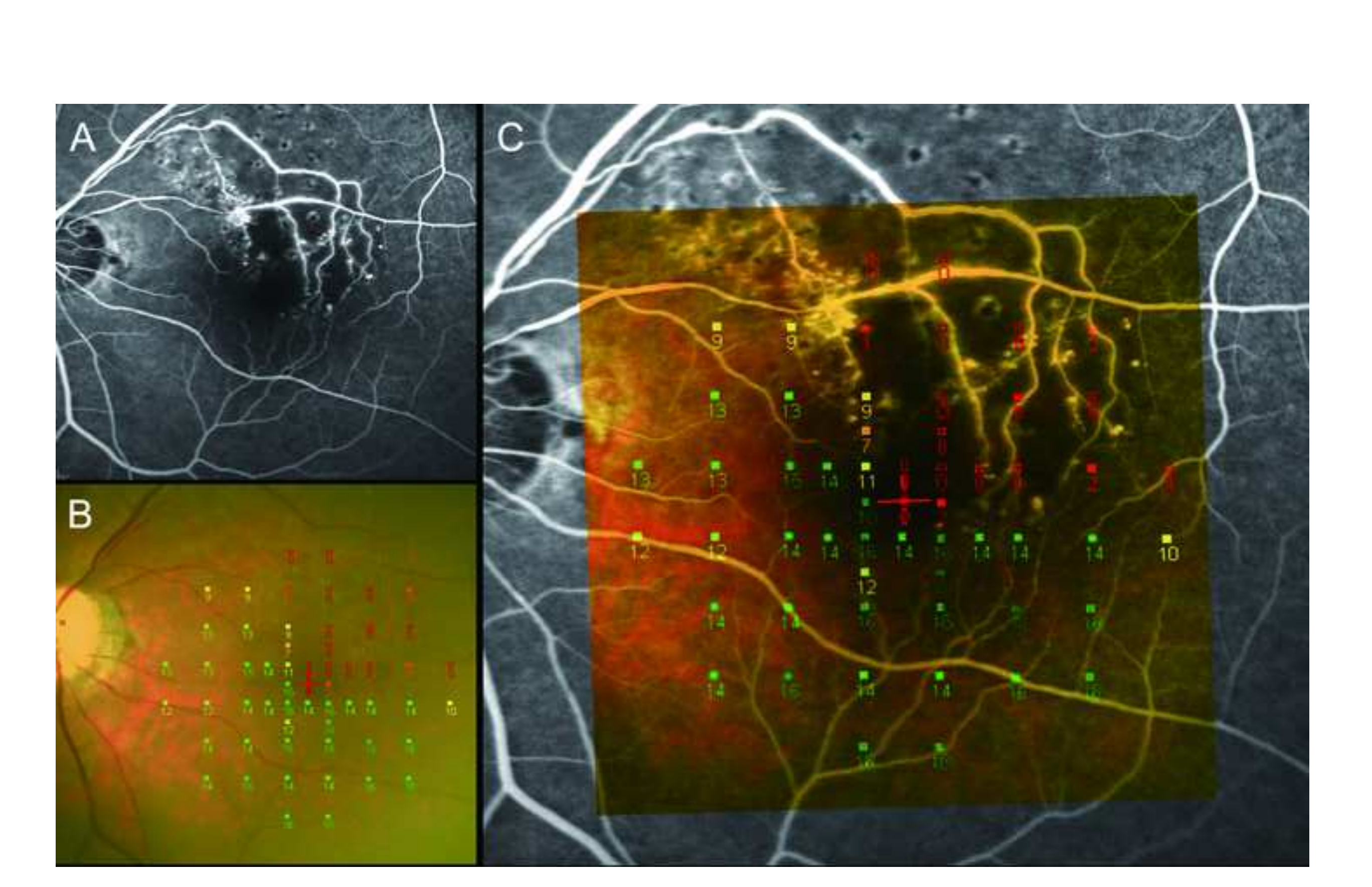

(1)

(1)

.

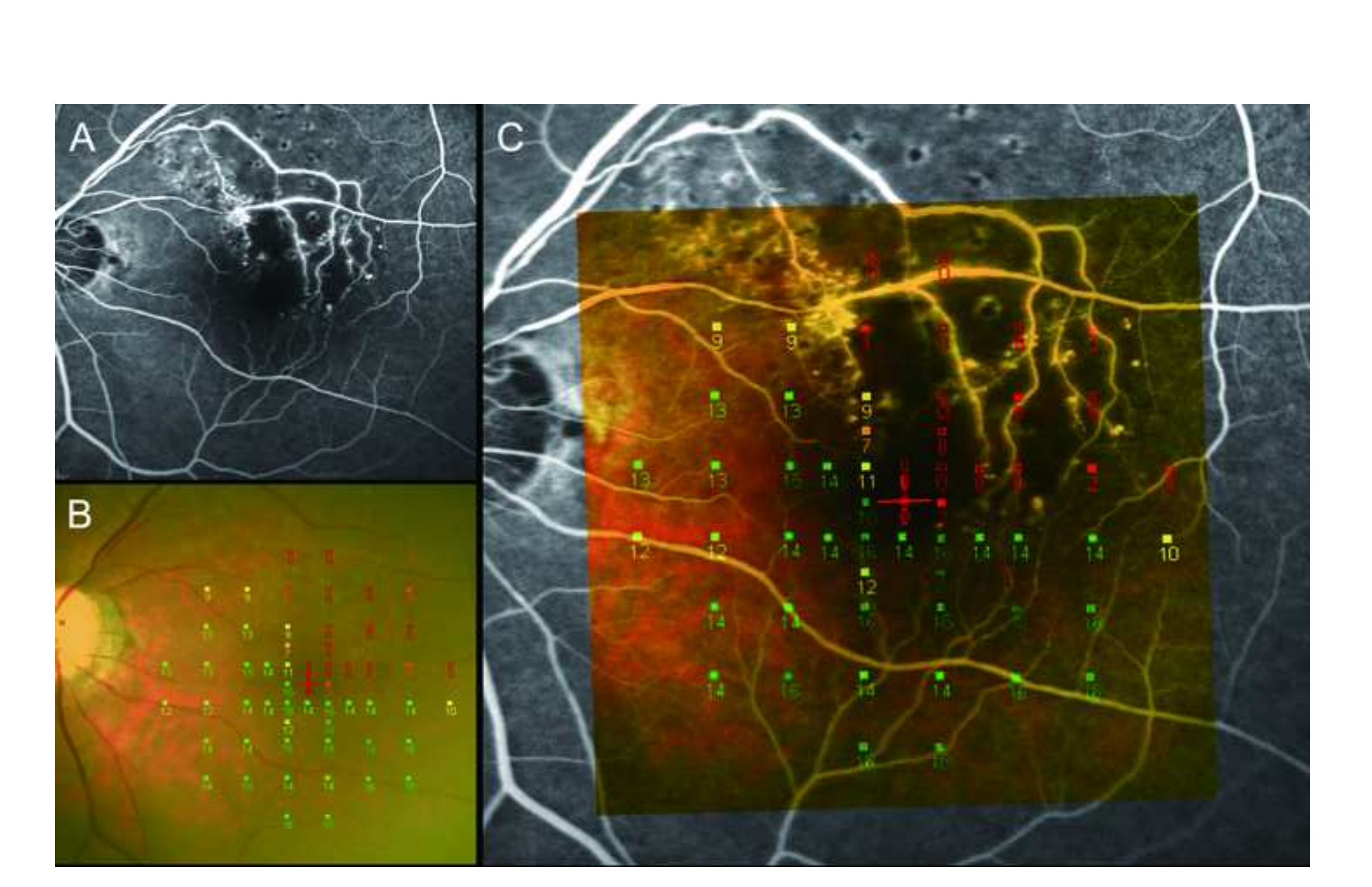

. 
Click here to download high resolution image
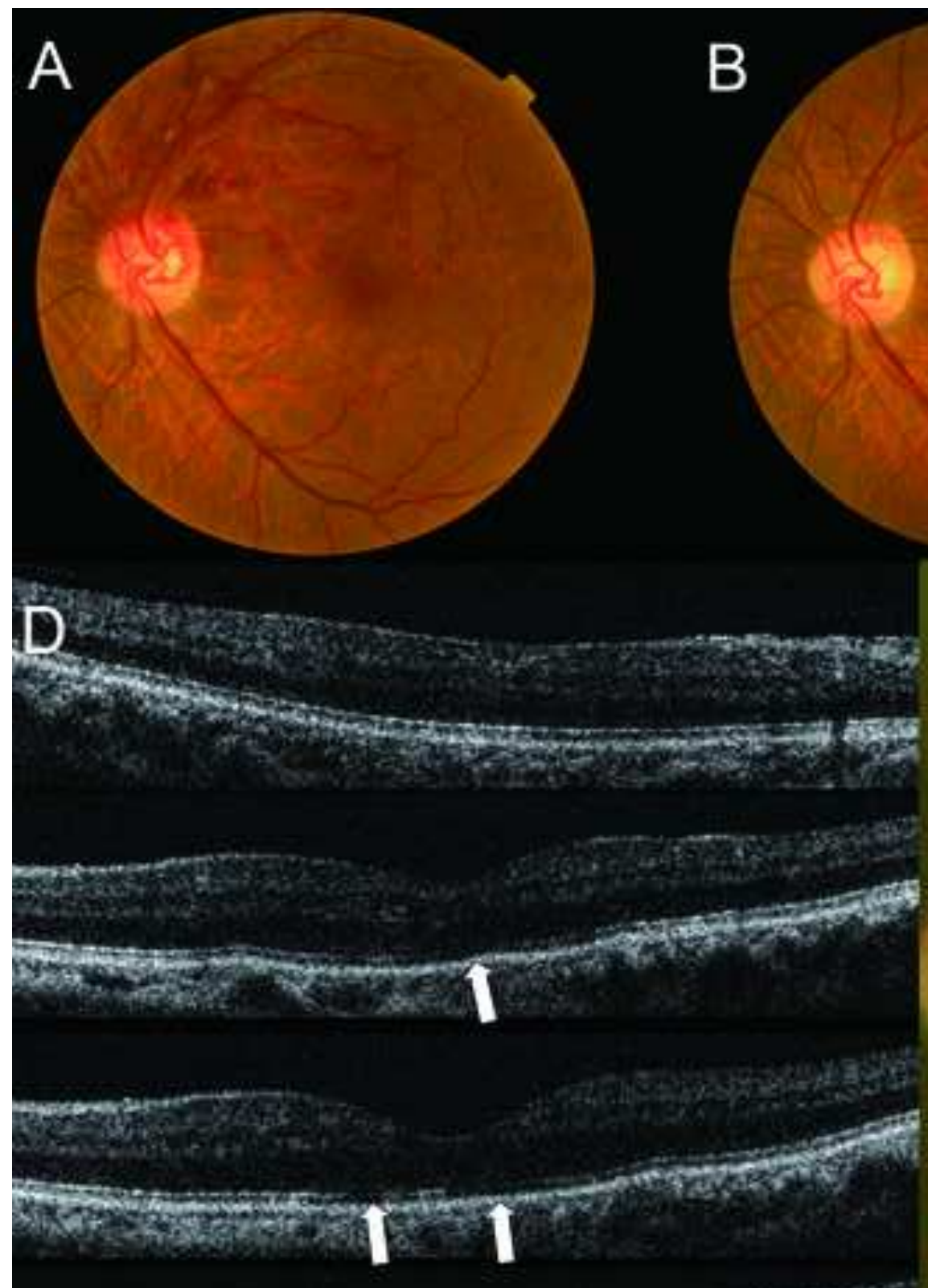

E

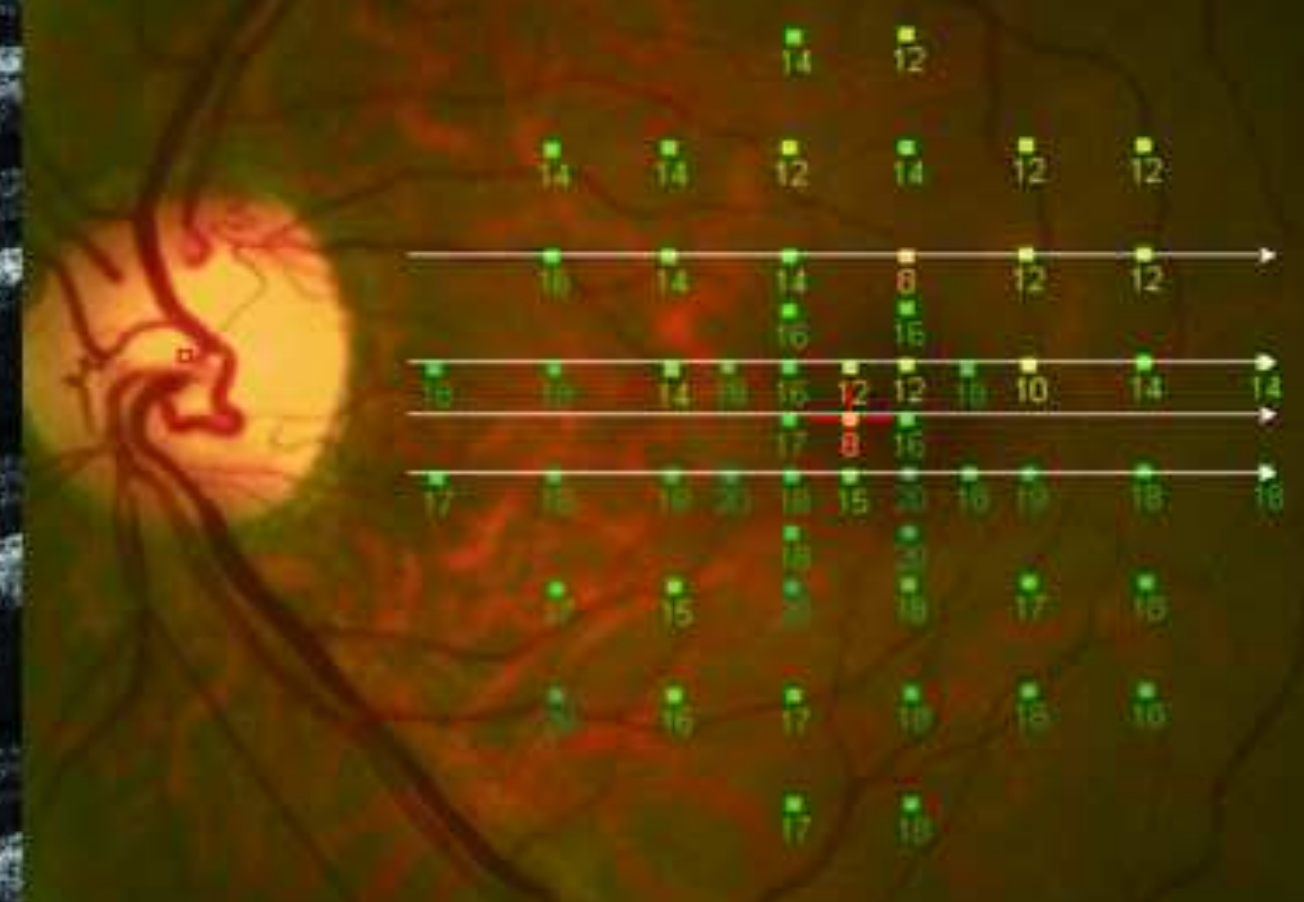

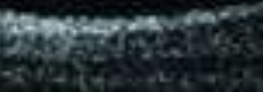

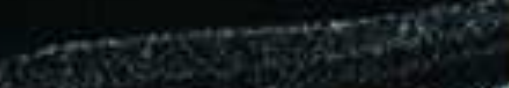

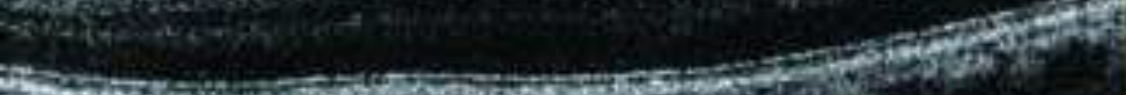

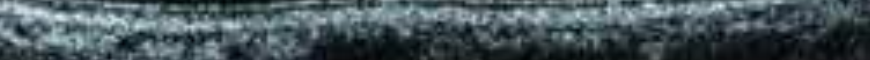

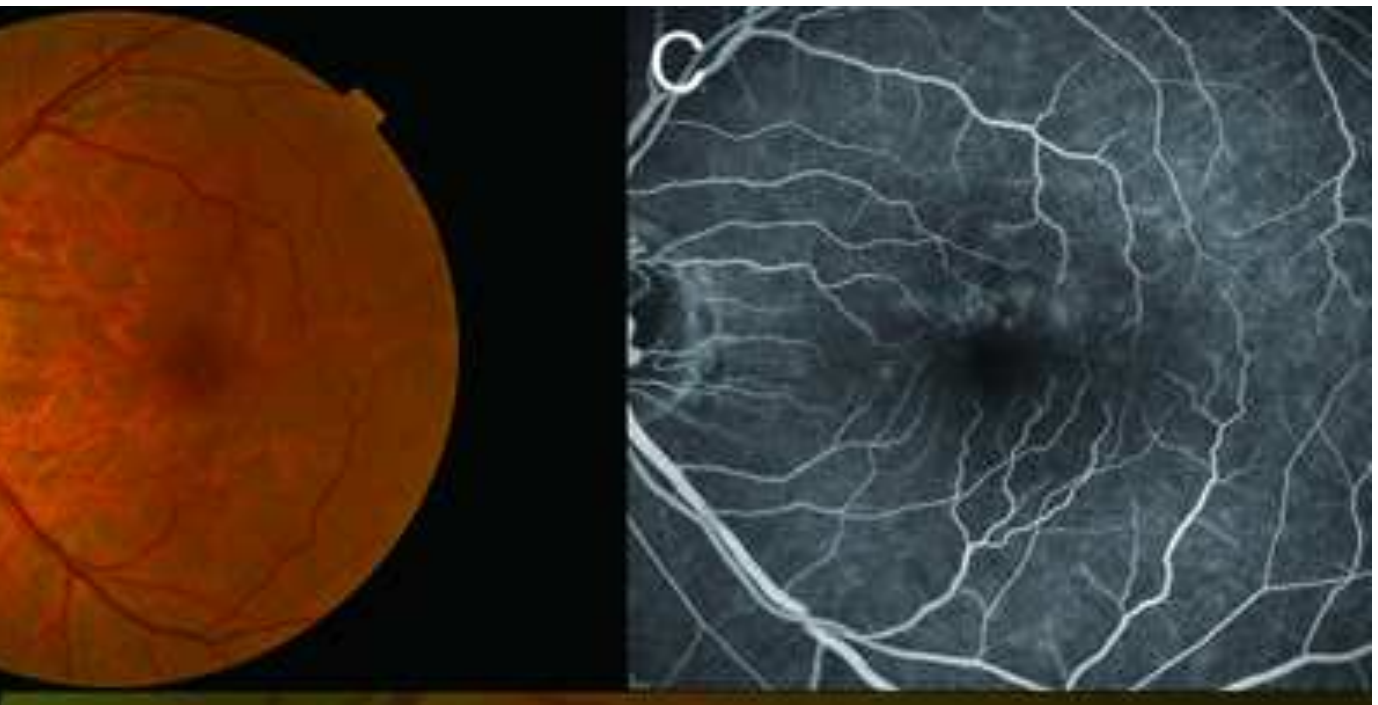



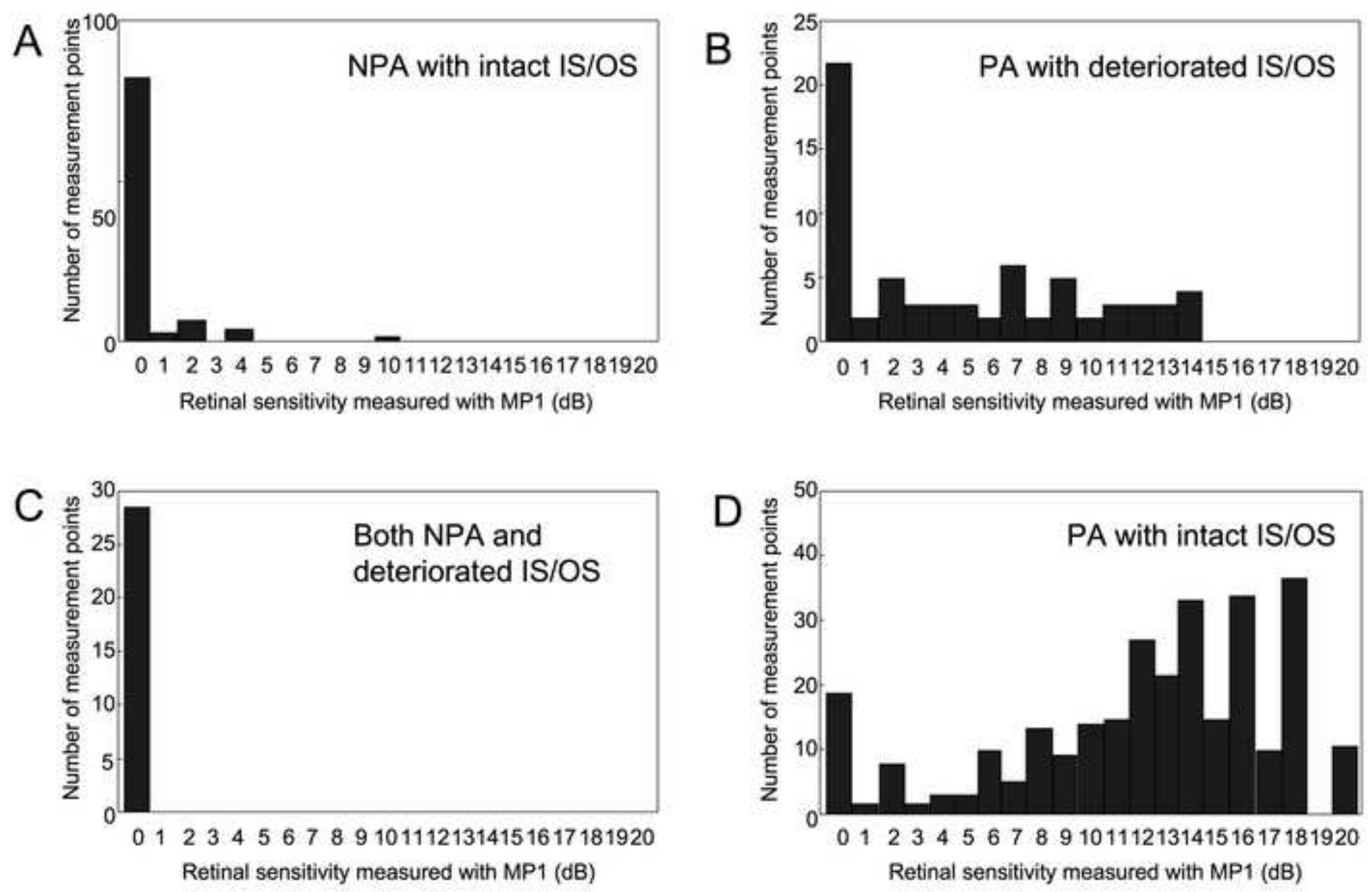